\title{
Identificación de parásitos gastrointestinales en un criadero de avestruces (Struthio camelus) del departamento del Cauca
}

Paz Campuzano, Juan; Rivera Calderón, Luis; Tigreros Muñoz, Surelly; Acosta Jurado, Ana; Sarmiento Vela, Jhon; Valencia Hoyos, Carlos; Castro Castro, Fernando

Identificación de parásitos gastrointestinales en un criadero de avestruces (Struthio camelus) del departamento del Cauca

Revista Colombiana de Ciencia Animal, vol. 11, núm. 1, 2019

Universidad de Sucre, Colombia

DOI: https://doi.org/10.24188/recia.v0.n0.2019.698

Los autores permiten a RECIA reimprimir el material publicado en él. En caso de que un autor quiera traducir o usar una publicación parcial o completa de nuestro Diario, el autor debe obtener un permiso por escrito del editor de la revista.

Esta obra está bajo una Licencia Creative Commons Atribución 4.0 Internacional. 


\section{Identificación de parásitos gastrointestinales en un criadero de avestruces (Strutbio camelus) del departamento del Cauca}

Identification of gastrointestinal parasites in a hatchery of ostrich (Struthio camelus) in the Cauca state

Juan Paz Campuzano

DOI: https://doi.org/10.24188/recia.v0.n0.2019.698

Universidad Antonio Nariño, Facultad de Medicina

Veterinaria y Zootecnia, Popayán, Cauca, Colombia

juapaz@uan.edu.co

(iD http://orcid.org/0000-0003-4216-2182

Recepción: 01 Febrero 2019

Aprobación: 15 Abril 2019

Luis Rivera Calderón

Publicación: 30 Abril 2019

Universidad Antonio Nariño, Facultad de Medicina Veterinaria y Zootecnia, Popayán, Cauca, Colombia lgriveramvz@gmail.com

(iD http://orcid.org/0000-0003-4455-8404

Surelly Tigreros Muñoz

Zootecnista Independiente, Colombia

surellyzoo@gmail.com

iD http://orcid.org/0000-0003-2998-2717

Ana Acosta Jurado

Universidad Antonio Nariño, Facultad de Medicina

Veterinaria y Zootecnia, Popayán, Cauca, Colombia

ana.acostado@uan.edu.co

iD http://orcid.org/0000-0001-9238-4432

Jhon Sarmiento Vela

Universidad Antonio Nariño, Facultad de Ingenieria Biomédica, Popayán, Cauca, Colombia

jfsarmiento@uan.edu.co

(iD http://orcid.org/0000-0001-9791-8173

Carlos Valencia Hoyos

Universidad Antonio Nariño, Facultad de Medicina

Veterinaria y Zootecnia, Popayán, Cauca, Colombia valencia@uan.edu.co

(iD) http://orcid.org/0000-0002-0970-1589

Fernando Castro Castro

Universidad Antonio Nariño, Facultad de Medicina

Veterinaria y Zootecnia, Popayán, Cauca, Colombia

favian.castro@uan.edu.co

(iD http://orcid.org/0000-0002-9016-1814 


\section{RESUMEN:}

Los avestruces poseen características productivas que la hacen una especie atractiva para el mercado de carne y huevos, además de otros subproductos como plumas, grasas, y piel. En diferentes países del mundo, incluyendo a Colombia, la producción de esta especie está aumentando y para mantener su población en cautiverio se están tomando medidas preventivas contra enfermedades virales, bacterianas y parasitarias. El objetivo de este trabajo fue identificar parásitos gastrointestinales en un criadero de avestruces del departamento del Cauca. Para el estudio, se colectó material coprológico de 38 animales, 19 adultos (> 7 años de edad), 9 Juveniles ( 1 año), 10 polluelos (1 mes), siendo enviado a $4{ }^{\circ} \mathrm{C}$ a un laboratorio donde fue procesado y examinado. Se identificaron quistes compatibles con Eimeria sp. (40\%), Balantidium sp. (33,3\%), Entamoeba sp. (13,3\%) y huevos compatibles con Libyostrongylus sp. (13,3\%), no hubo correlación entre las edades y la carga parasitaria, así como, el sexo y la carga parasitaria. A pesar de que los animales muestreados no presentaron signos clínicos de enfermedad parasitaria, es necesario realizar más estudios para comprender el ciclo biológico de estos parásitos en los avestruces de la región, en el presente trabajo se sugiere la presencia de Libyostrongylus sp., el cual ya ha sido reportado una vez en Colombia.

Palabras Clave: Ratites, cautiverio, protozoarios, nematodos, coprológico, enfermedad, ciclo biológico.

\section{Abstract:}

The ostriches have attractive productive characteristics for the market of meat and eggs, as well as by-products such as feathers, fats, and skin. In Colombia the production of this species is increasing and to maintain its population in captivity, preventive measures are being taken against viral, bacterial and parasitic diseases. The objective of this study is to identify gastrointestinal parasites in an ostrich hatchery in the Cauca state. Coprological material was collected from 38 animals, 19 Adults ( $>7$ years of age), 9 young (1 year), 10 chicks ( 1 month), this material was sent at $4{ }^{\circ} \mathrm{C}$ to be processed and examined in the laboratory. Were identified cysts compatibles with Eimeria sp. (40\%) Balantidium sp. (33.3\%), Entamoeba sp. (13,3\%) and eggs compatibles with Libyostrongylus sp. (13.3\%), there was no correlation between ages and parasitic load; also there was no correlation between sex and parasitic load. Although the animals sampled did not present clinical signs of parasitic disease, it is necessary to carry out further studies to understand the biological cycle of these parasites in ostrichs of this region, in the present study we suggested the presence of Libyostrongylus sp., which has been reported once in Colombia.

KEYWORDS: Ratites, captivity, protozoans, nematodes, coprological, disease, biologic cycle.

\section{INTRODUCCIÓN}

El avestruz (Struthio camelus) es un ave herbívora, diurna, que en estado natural se observa en grupos nómadas de 5 a 50 individuos, movilizándose con otros animales de pastoreo, incluidas la cebra, el antílope y la jirafa. Alcanzan su madurez sexual a los 4-5 años de edad con un peso de $145 \mathrm{~kg}$ y poseen una longevidad de $30 \mathrm{a}$ 40 años (1). Las hembras adultas llegan a poner de 27 a 36 huevos por año, con un peso promedio de 1500 g. Estos huevos cuentan con valores nutricionales similares a los de otras aves y su cáscara también puede ser usada con fines artesanales $(2,3)$.

Las características productivas y reproductivas mencionadas anteriormente hacen del avestruz una especie atractiva para el mercado de carne y huevos, además de otros subproductos como plumas, aceites y piel $(3,4)$. En diversos países de Sudamérica, incluyendo Colombia, la producción de esta especie está aumentando $(5,6)$. En Colombia, la especie se encuentra bien establecida en los departamentos del Meta, Cundinamarca, Boyacá, Cauca, Valle del Cauca y Quindío (6). A pesar de las medidas sanitarias para evitar el surgimiento de enfermedades en los avestruces, se ha reportado que algunos agentes infecciosos incluyendo virus, bacterias y parásitos gastrointestinales, continúan siendo un problema para los criadores de esta especie $(7,8,9)$. Por este motivo, el objetivo de este trabajo fue identificar la presencia de parásitos gastrointestinales en un criadero de avestruces del departamento del Cauca.

\section{MATERIALES Y MÉTODOS}

Área de estudio. El experimento se realizó en un criadero de avestruces localizado en el municipio de Puerto Tejada en el norte del departamento del Cauca, Colombia, el cual posee una topografía plana y homogénea, 
con un promedio de 970 m.s.n.m. La temperatura media del área de estudio fue de $24^{\circ} \mathrm{C}$, con precipitaciones pluviales entre 1000 - $2000 \mathrm{~mm}$ y humedad del 70 al $90 \%$.

Animales de estudio. Se estudiaron 38 avestruces de cuello rojo (Struthio camelus camelus), 19 Adultos (> 7 años de edad) (Figura 1A), 9 Juveniles (de 1 año), 10 polluelos (de 1 mes) (Figura 1B). De estos fue posible determinar el sexo en los adultos y los juveniles (13 hembras y 15 machos). Los individuos se alojaban en corrales clasificados de acuerdo a la edad y estado reproductivo, siendo alimentados con diversas especies de plantas como: King grass (Pennisetum purpureum), nacedero (Trichantheragigantea), matarratón (Gliricidia sepium) y maní forrajero (Arachis pintoi).

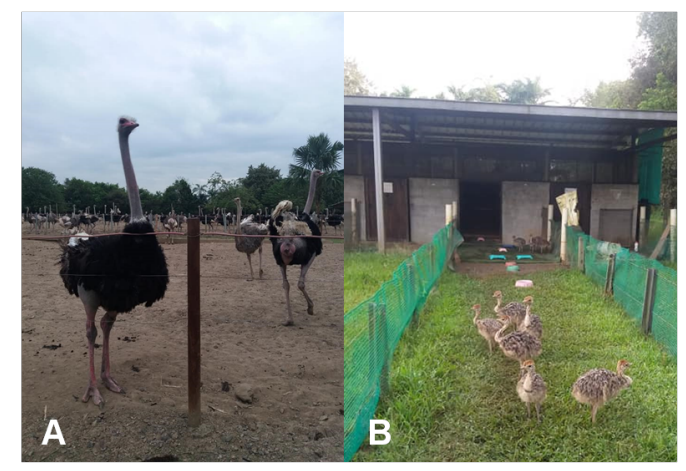

FIGURA 1

Avestruces del criadero en el municipio de Puerto Tejada. A. Avestruces en corral de 1 año. B. Polluelos de avestruz de un mes de edad.

Manejo y muestreo de los animales. El manejo de los animales adultos y jóvenes en los corrales de confinamiento fue realizado con la ayuda de personal calificado. Durante la colecta, los individuos no fueron capturados, por lo que no sufrieron estrés. El muestreo coprológico se efectuó 30 minutos después de suministrar el primer alimento de la mañana. Se obtuvo 2 gramos de materia fecal por cada animal, evitando el contacto con el suelo.

Análisis de laboratorio. Después de colectadas y rotuladas, las muestras se depositaron en una nevera a $4^{\circ} \mathrm{C}$ y se transportaron para el laboratorio de diagnóstico veterinario (Zoolavet). En el laboratorio se utilizaron dos métodos para la identificación de parásitos: El método directo en solución salina y lugol descrito por la OMS (10) y Campo et al (11), además del método de flotación con solución salina saturada (12).

Análisis estadístico. Se utilizó un análisis descriptivo para determinar las frecuencias y distribuciones muestreales de parásitos gastrointestinales relacionadas con las variables edad y sexo. Adicionalmente, se empleó la prueba de independencia del Chi-cuadrado para determinar si existían diferencias significativas en cuanto a la proporción de animales parasitados independiente del sexo y edad. Estadística significativa fue considerada con $\mathrm{p}<0.05$.

\section{RESULTADOS}

De los 38 avestruces estudiados 15 fueron positivos para parásitos ( 9 eran machos, 4 hembras y 2 polluelos). Se identificaron quistes compatibles con Eimeria sp., (40\%), Balantidium sp., (33,3\%), Entamoeba sp., (13,3\%) (Figura 2A), además de huevos compatibles con Libyostrongylus sp., (13,3\%) (Figura 2B). En la tabla 1 se encuentran los parásitos gastrointestinales en los avestruces según su edad y sexo. 


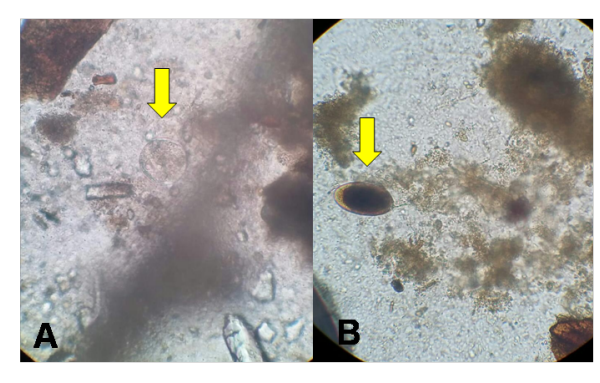

FIGURA 2

Quistes y huevos parasitarios observados en heces de los avestruces. A.

Quiste de Balantidium sp. B. Sugestivos de huevo de Libyostrongylus sp.

TABLA 1

Identificación de parásitos gastrointestinales en los avestruces

\begin{tabular}{|c|c|c|c|}
\hline $\begin{array}{c}\text { No. } \\
\text { Muestra }\end{array}$ & Edad & Sexo & Parasito \\
\hline 1 & 1 año & macho & $\begin{array}{l}\text { Compatible con Quistes de } \\
\text { Eimeria sp. }\end{array}$ \\
\hline 2 & 1 aก๊̃o & macho & $\begin{array}{l}\text { Compatible con Quistes de } \\
\text { Entamoeba sp. }\end{array}$ \\
\hline 3 & 1 año & macho & $\begin{array}{l}\text { Compatible con Quistes de } \\
\text { Balantidium sp. }\end{array}$ \\
\hline 4 & 1 aก̃o & macho & $\begin{array}{l}\text { Compatible con Quistes de } \\
\text { Balantidium sp. }\end{array}$ \\
\hline 5 & 7 años & macho & $\begin{array}{l}\text { Compatible con Quistes de } \\
\text { Eimeria sp. }\end{array}$ \\
\hline 6 & 7 años & macho & $\begin{array}{l}\text { Compatible con Quistes de } \\
\text { Balantidium sp. }\end{array}$ \\
\hline 7 & 7 años & macho & $\begin{array}{l}\text { Compatible con Quistes de } \\
\text { Eimeria Sp. }\end{array}$ \\
\hline 8 & 7 años & macho & $\begin{array}{l}\text { Huevos Sugestivos de } \\
\text { Libyostrongylus sp. }\end{array}$ \\
\hline 9 & 1 aก̃o & hembra & $\begin{array}{l}\text { Compatible con Quistes de } \\
\text { Balantidium sp. }\end{array}$ \\
\hline 10 & 1 aก̃o & hembra & $\begin{array}{l}\text { Compatible con Quistes de } \\
\text { Entamoeba sp. }\end{array}$ \\
\hline 11 & 7 años & hembra & $\begin{array}{l}\text { Huevos sugestivos de } \\
\text { Libyostrongylus sp. }\end{array}$ \\
\hline 12 & 7 años & hembra & $\begin{array}{l}\text { Compatible con Quistes de } \\
\text { Balantidium sp }\end{array}$ \\
\hline 13 & 7 años & hembra & $\begin{array}{l}\text { Compatible con Quistes de } \\
\text { Eimeria Sp. }\end{array}$ \\
\hline 14 & 28 dias & ND & $\begin{array}{l}\text { Compatible con Quistes de } \\
\text { Eimeria Sp. }\end{array}$ \\
\hline 15 & 28 días & ND & $\begin{array}{l}\text { Compatible con Quistes de } \\
\text { Eimeria Sp. }\end{array}$ \\
\hline
\end{tabular}

Con la prueba de chi-cuadrado se identificó que no existen diferencia en cuanto a la proporción de animales parasitados independiente de la edad ( $p>0,05)$, el valor de chi-cuadrado fue de 4,428 y los grados de libertad 3 , por lo cual se rechaza la hipótesis nula que propone una distribución similar en la presencia parasitaria independiente de la edad (Figura 3A). De la misma forma, no se observó diferencia en función del sexo ( $p>0.05$ ), el valor de chi-cuadrado fue de 0,619 y los grados de libertad 2, siendo rechazada la hipótesis nula que plantea una distribución similar en la presencia parasitaria independiente del sexo (Figura 3B). 


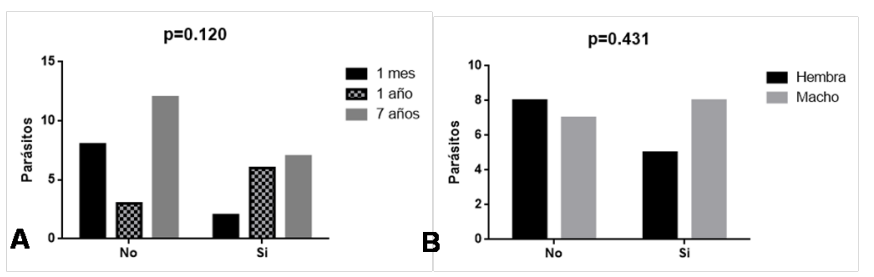

FIGURA 3

Distribuciones muestréales de parásitos gastrointestinales relacionadas con las variables edad (A) y sexo (B).

\section{DISCUSIÓN}

En los avestruces del presente trabajo se identificaron algunos quistes de protozoarios compatibles con: Balantidium sp., Entamoeba sp., y Eimeria sp. Ponce-gordo et al (13) también encontraron Balantidium sp, sin signos clínicos asociados. Estos autores sugieren que el ciclo biológico del género Entamoeba sp, puede desarrollarse tanto en avestruces silvestres como en humanos que se encuentran en contacto permanente. Además, en un estudio anterior realizado por el mismo grupo, se apreciaron quistes de protozoarios en avestruces compatibles con: Balantidium sp., y Eimeria sp., con una prevalencia baja comparada con otros protozoarios como: Trichomonas gallinae, Tetratrichomonas gallinarum (14), en contraste con los resultados de este trabajo. Como menciona Ponce Gordo (14), los diferentes protozoarios que se han encontrado en Rheas y Ostriches, pueden estar compartidos con otras aves y mamíferos (incluyendo el hombre), esto hace difícil establecer su especificidad por alguna especie y su ciclo biológico completo. Por lo tanto son necesarios más estudios para entender la interacción entre estos parásitos y el huésped.

Los países importadores de avestruces y sus productos poseen regulaciones veterinarias para evitar el ingreso de agentes patógenos, incluyendo parásitos, que pongan en riesgo su salud pública y sus granjas de avestruces, sin embargo, diferentes parasitos exóticos se han reportado en sus producciones de ratites con un impacto económico poco esclarecido $(14,16)$.

En el estudio actual, se observaron huevos compatibles con el nematodo Libyostrongylus sp. Algunos autores han reportado la presencia de este género en diferentes países de América como: México, Brasil y Colombia $(6,15,16)$. Sin embargo, el análisis de las muestras mediante la técnica de flotación, no permite afirmar el diagnóstico definitivo de los huevos de estos parásitos, debido a que este género es indistinguible del nematodo Codiostomum struthionis. Además sus larvas también poseen una morfología similar por lo que la técnica recomendada para su clasificación es el coprocultivo $(6,7,17)$.

Los parásitos gastrointestinales Codiostomum sp., y Libyostrongylus sp., pueden producir signos clínicos en diferentes edades de los avestruces $(7,18)$. Algunos autores hacen referencia al parasitismo por esta especie de nematodo, demostrando que las altas tasas de morbilidad y mortalidad se encuentran en animales jóvenes, teniendo un impacto mayor en las aves de hasta 3 meses de edad (50\%), mientras que en avestruces adultas la tasa de mortalidad es menor al 10\%, y en ocasiones no manifiestan signos clínicos a pesar de poseer cargas parasitarias sean variables (6). Aunque en el estudio no se apreció las cargas parasitarias de los individuos, los avestruces afectados por el Libyostrongylus sp., no presentaban ninguna sintomatología clínica en el criadero, esto se puede explicar debido a que los animales se encontraban en su etapa adulta. Además no se observó diferencia en cuanto a la distribución de animales parasitados independiente del sexo.

Futuros estudios deben ser elaborados para comprender el ciclo biológico de parásitos gastrointestinales en ratites de la región, en este trabajo se sugiere la presencia de Libyostrongylus sp., el cual ya ha sido reportado una vez en Colombia por Mariño-Gonzales et al (6) en el año de 2017.

Adicionalmente, se recomienda que todos los animales nuevos que ingresen al criadero se sometan a un período de cuarentena, con tratamiento antiparasitario interno y externo, además de la aplicación de vacunas 
requeridas en la región. Es ideal separa a los animales por edad y evitar el ingreso de fauna silvestre o animales domésticos que puedan contaminar el ambiente, producir estrés y transmitir enfermedades a los ratites.

Los criadores deben realizar exámenes coprológicos periódicos y determinar el tratamiento antiparasitario ideal para prevenir y controlar enfermedades en la granja. Finalmente, los animales deben contar con agua potable y una dieta inocua de acuerdo a su estado productivo y reproductivo, que disminuyan el riesgo de parasitosis.

\section{Conflicto de interés}

Los autores declaran que no tienen conflictos de interés con respecto al trabajo presentado en este informe.

\section{REFERENCIAS}

1. Cooper RG, Horbańczuk JO, Villegas-Vizcaíno R, Kennou Sebei S, Faki Mohammed AE, Mahrose KM. Wild ostrich (Struthio camelus) ecology and physiology.Trop Anim Health Prod. 2010. 42(3):363-73

2. Al-Obaidi F, Al-Shadeedi S. Comparison study of egg morphology, component and chemical composition of ostrich, emu and native chickens. J. Genetic. Environ. Reour. Conserv. 2015, 3(2):132-137.

3. Bouyeh M, Seidavy A, Mohammadi H, Sahoo A, Laudadio V, Tufarelli V. Effect of climate region and stocking density on ostrich(Struthio camelus) productive performances. Reprod Domest Anim. 2017; 52(1):44-48. https://doi.org/10.1111/rda.12793

4. American Ostrich Association (AOA). The Ostrich Industry 2.0 presentation.2019. [Citado 28 de enero de 2019]. URL Disponible en: https://www.ostriches.org/ostrich-industry-2-0-presentation

5. World Ostrich Association (WOA). World Ostrich Production Statistics Newsletter No. 19.2014. [Citado 28 de enero de 2019]. URL Disponible en: http://world-ostrich.org/world-ostrich-production-statistics/

6.Mariño-González GA, Ramírez-Hernández A, Cortés-Vecino JA. Libyostrongylus douglassii (Strongylida: Trichostrongylidae) in ostrich (Struthio camelus) farms from Colombia. Vet Parasitol. 2017; 235:253-56.

7.De Oliveira FC, Ederli NB, Lopes CW, Rodrigues M de L. Pathological findings in the caeca of naturally infected ostriches, Struthio camelus Linnaeus, 1758 (Aves, Struthionidae) parasitized by Codiostomum struthionis (Horst, 1885) Railliet and Henry, 1911 (Nematoda, Strongylidae). Vet Parasitol. 2009; 165(1-2):175-178. https://doi.org/10.1016/j.vetpar.2009.06.034

8.Ruenphet S, Satoh K, Tsujimura M, Hasegawa T, Takehara K. Strategies of newcastle disease vaccination for commercial ostrich farms in Japan. J Vet Med Sci. 2012; 74(7):905-908. https://doi.org/10.1292/jvms.11-0502

9.Keokilwe L, Olivier A, Burger WP, Joubert H, Venter EH, Morar-Leather D. Bacterial enteritis in ostrich (Struthio camelus) chicks in the western cape province, South Africa. Poult Sci. 2015; 94(6):1177-1183. https:// doi.org/10.3382/ps/pev084

10. OMS. Métodos básicos de laboratorio en parasitología médica. World Health Organization; 1992. http:// apps.who.int/bookorders/anglais/detart 1 .jsp?codlan $=3 \& \operatorname{cod}$ col $=15 \& \operatorname{cod} c \mathrm{ch}=354$

11. Campo LF, Botero LE, Guitiérrez LA, Cardona JA. Reproducibilidad del examen directo de heces y de la concentración formol-éter y validez del examen directo de heces para el diagnóstico de parásitos intestinales. Archivos de Medicina. 2015; 11(4):1-9. https://doi.org/10.1016/s0025-7753(02)72402-0

12.Figueroa-Castillo JA, Jasso-Villazul C, Liébano-Hernández E, Martínez-Labat P, Rodríguez-Vivas RI, ZárateRamos, JJ. Examen coproparasitoscópico. En: Rodríguez-Vivas R.I. (Editor). Técnicas para el diagnóstico de parásitos con importancia en salud pública y veterinaria. 1 edición. México, D.F. AMPAVE-CONASA. 2015.

13.Ponce-Gordo F, Jiménez-Ruiz E, Martínez-Díaz RA. Tentative identification of the species of Balantidium from ostriches (Struthio camelus) as Balantidiumcoli-like by analysis of polymorphic DNA. Vet Parasitol. 2008;157(1-2):41-49. https://doi.org/10.1016/j.vetpar.2008.06.024

14.Ponce Gordo F, Herrera S, Castro AT, García Durán B, Martínez Díaz RA.Parasites from farmed ostriches (Struthio camelus) and rheas (Rhea americana) in Europe. Vet Parasitol. 2002; 107(1-2):137-160. https:// doi.org/10.1016/s0304-4017(02)00104-8 
15.Lelis RT, de Andrade JG, Vieira RA, Da Matta RA.Population dynamics of Libyostrongylus dentatus and L. douglassii of ostriches raised in the Northern Rio de Janeiro State, Brazil. Vet Parasitol. 2014; 200(1-2):147-152. https://doi.org/10.1016/j.vetpar.2013.11.022

16. Sánchez-Ayala JR., Cruz-Mendoza I, Figueroa-Castillo JA, Vital-García C. First report of Libyostrongylus douglassii (Strongylida: Trichostrongylidae) in ostriches (Struthio camelus) from Mexico. Vet Parasito. 2018; 12:31-34. https://doi.org/10.1016/j.vprsr.2018.01.007

17. Ederli NB, de Oliveira, FC. Comparative morphology of the species of Libyostrongylus and Codiostomum, parasites from ostriches, Struthio camelus, with a identification key to the species. Braz. J. Vet. Parasitol. 2014; 23(3):291-300. https://doi.org/10.1590/s1984-29612014061

18. De Andrade JG, Lelis RT, Da Matta RA. Occurrence of nematodes and anthelmintic management of ostrich farms from different Brazilian states: Libyostrongylus douglassii dominates mixed infections. Vet Parasitol. 2011; 178:129-133. https://doi.org/10.1016/j.vetpar.2010.12.032

Los autores permiten a RECIA reimprimir el material publicado en él. En caso de que un autor quiera traducir o usar una publicación parcial o completa de nuestro Diario, el autor debe obtener un permiso por escrito del editor de la revista.

CC BY 